\title{
Twenty Questions for PER: How Does It All Fit Together?
}

\author{
Edward F. Redish*, Michael C. Wittmann† \\ *Department of Physics, University of Maryland, College Park, MD 20742-4111, USA \\ $\dagger$ Department of Physics and Astronomy, University of Maine, Orono, ME 04469-5709, USA
}

\begin{abstract}
In physics education research (PER), for many years now we have focused our attentions on finding ways to improve our instruction and have achieved some notable successes. In this paper, we suggest that the time has come to embed this activity in a more complete and scientific view of PER, one that builds a coherent understanding of the system of teaching and learning in addition to improving the practice of our instruction. We outline five broad topics of interest for PER and discuss questions that need to be addressed in each topic over the next few years. The topics are: the model of the participants, the model of the contexts, the model of the content, the engineering of instruction, and the overall epistemology of PER - How do we decide when we think we know something?
\end{abstract}

\section{INTRODUCTION}

Little of what we do in physics education research (PER) resembles the complex and productive interplay of theory and experiment common to other sciences. To promote such an interplay, we suggest some broad general questions to help put research in PER in perspective. Our goal is to suggest paths of action and dialogues within disparate research areas.

We often say that as physics education researchers, we are applying the methods of science to help us understand how our students learn and do not learn physics. Often, however, we restrict our "application of the scientific method" to observing what our students do and trying to correlate their learning with instructional changes that seem, intuitively, to make sense to us. It is more like "seat-of-the-pants" engineering than like physics; it is more like Edison's search for the proper filament for a light bulb than like the current attempt to understand spintronics in order to (some time in the future) build a better microchip.

In scientific research, experiment and theory perform an intricate dance, with theory taking the lead at one time and suggesting experiments, with experiment taking the lead at another time and producing results that demand new theoretical explanations. A good example of this is the development of the theory of electricity. Although Benjamin Franklin was much concerned with saving houses from lightning strikes, his research into electricity involved much more than engineering better lightning rods. He did extensive ex- periments and proposed theories of the structure of electricity, including demonstrating the two charge model and proposing the theory of charge conservation. Coulomb's later detailed and accurate experiments relied heavily on the theoretical two-charge model built up as a result of earlier careful experimental and theoretical developments.[1]

In PER, we rarely use a serious theoretical frame. We are unable to generate reliable predictions in situations we haven't observed, and we are often unable to understand what our experimental results are telling us about theory. To move forward into a more productive and scientific mode of research, we must understand and model complicated educational systems and situations. We need to model the participants, both students and teachers. We need to model the way they interact, both with their overall environment and with the specific environments that happen in their classrooms. We need to model the content they are learning, both physically and pedagogically. Clearly, these models of participants, contexts, and content are not independent; they interact strongly. Contexts play a crucial role in determining the structure and response of individuals. The properties and responses of individuals play a crucial role in determining how group interactions take place. The way individuals think determines how content is seen, organized, and learned (and even discovered).

These three strongly interacting research areas suggest that our theories need to overlap physics, cogni- 
tive, and behavioral science. In each of these areas, much is known both inside and outside the PER community. We need to consider what we can learn from successful engineering of instructional environments and how our models of participants, contexts, and content can inform the engineering of our classrooms. In this brief paper, we will not summarize what is known in any of these areas. Rather, we will raise some broad general questions that appear to us to be a useful guide to synthesis in PER.

\section{PER: WHAT DO WE KNOW AND HOW DO WE KNOW IT?}

Our first question concerns the epistemology of PER: How do we decide when we know something? In physics, we are accustomed to having mathematical predictions and quantitative experiments. People, like individual electrons, appear to have free will; people's behavior is predictable only statistically. But people, unlike electrons, are not identical or interchangeable. This leads to numerous questions of "How do we know? Why do we believe?"

The broad question for this section we might call: The Epistemology Question - What are appropriate tools, qualitative and quantitative, for deciding we have learned something in PER?

There are many questions one could ask to determine how we decide to know something in PER. The first comes from the fact that we listen to our students, interview them, and monitor their behavior on homework, quizzes, and tests. What do we learn from this?

1. What information do we need in order to infer what's happening in an individual's mind?

If we are to follow the route suggested by scientific progress and to try to make any sense of student thinking in terms of mechanisms, those mechanisms are likely to be inferred rather than observed. Inferring what students and teachers are thinking from their behavior only expresses a part of what's happening. It's rather like inferring quarks inside a nucleon. How do we decide if a mental model or a social response is "really there"? Does it suffice to invoke Gell-Mann's early hedge and say "It doesn't matter if it's really there or not, this hypothesis is a useful way of thinking about the phenomena we are trying to describe"? What experimental data and triangulations do we need to decide a mental description is reliable and useful?

Other questions probe the issue of bridging the study of one student and generalizing to a class and considering hidden assumptions in statistical studies.
2. How many students do we need to study before we can be satisfied that we have seen "most of" the behaviors we can expect from a large population?

3. When do large-scale statistical studies give us appropriate information about the learning of individuals and when do they mislead us?

As physicists, we tend to prefer large-scale studies where we can use our familiar mathematical tools to generate numbers representing our uncertainty or error. But these tools and our interpretation of the results rely heavily on the underlying assumption that we have a homogeneous population that is normally distributed. This is rarely the case. We hope the law of large numbers will save us, averaging over many fluctuations. In medical research, it does not suffice to say "this drug is successful in treating this disease in $99 \%$ of the cases" if in the other $1 \%$ of the cases the patients go into anaphylactic shock. We first have to identify subpopulations at risk before applying statistics.

New technologies, both experimental and theoretical, are beginning to probe how people think. Do these methods have value for us?

4. Do brain-scanning techniques (fMRI, MEG, PET, EEG) give us any useful information about mental structures relevant for PER?

5. Does modeling with networks, either in the small (neural networks attempting to model specific tasks) or in the large (statistical network analysis showing emergent structures) tell us anything useful about how to model thinking in physics?

\section{MODELING THE PARTICIPANTS}

Students and teachers are the "electrons" and "nuclei" of our many-body classroom system. If we want to understand how our classroom works, we must understand how our students and teachers "work" - just as if we want to understand how the properties of an atom arise, we have to understand the properties of electrons and nuclei. Note, however, that we learn about electrons and nuclei by studying them through interactions. The same is true of students and teachers. We can build models of how people think and respond, but how those thoughts interact with and are affected by outside events, both physical and socio-cultural, is an essential component of this study. Synthetic models of thinking appropriate for PER have begun to be formulated. See, for example [2].

We might call the broad question for this section: The Ontology Question-What are appropriate mental 
structures for describing how people think and learn? This might be divided into two: statics (description) and dynamics (change). Note, however, that all brain processes are dynamic, so even calling our descriptive questions "statics" is a bit misleading.

We pose three broad questions that refine the static and descriptive question: What are appropriate mental structures for describing how students think about and interact with their educational environment?

6. Some researchers have described students' naïve knowledge as robust, others as volatile. Does one description dominate pre-expert students in physics? If not, what kind of description is appropriate and when?

7. Some researchers have described students' knowledge as stored and recalled, others as generated on the spot dynamically depending on context. Does this make a difference for us? If so, how do we determine which is right and when?

8. Students often appear not to use in our classrooms knowledge that they know and use in other contexts. How can we describe, understand, and control this phenomenon?

Of course we want to not just describe how students think: we want to change how they think, i.e., we want them to learn. We refer to this as "learning dynamics" and pose three broad questions related to the question: How do mental structures change in response to experience, i.e., how do people learn?

9. What is the mechanism by which we make sense of things?

10. What level of mental structure is appropriate to use to describe learning in physics?

11. What does it mean to make a transition from a novice to an expert, and how do we describe this transition and the transition process?

\section{MODELING THE CONTEXT}

Individuals build their cognitive structures through their response to and interaction with both their physical and socio-cultural environment. Our understanding of an individual's cognition and learning has to dovetail with our understanding of both how their physical and the socio-cultural worlds function. We need to develop a descriptive understanding of how social environments like a classroom function and an understanding of how students respond to and are shaped by these environments. For a discussion and review of the literature on these issues see [3].

We might call the broad question for this topic: The Socio-Cultural Question - How do students' experiences and interactions with their environments affect what they bring in to the class and their responses to learning environments? Under this topic, we have both static (descriptive) and dynamic (learning) issues and several broad questions.

We pose three questions related to statics:

12. What are appropriate ways of thinking about and describing the functioning of a group of people such as a classroom or small group interaction?

13. What aspects of group interactions give us information about physical reasoning in the group?

14. What are appropriate mental structures for describing how individuals are affected by and affect an educational environment?

We pose three questions related to learning:

15. What are the feedback loops that connect individual actions, group dynamics, and larger scale environmental factors?

16. How do mental structures change in response to experience, i.e., how does context affect how people learn?

17. How does the learning of an individual and a group differ, and when do these differences matter?

\section{MODELING THE CONTENT}

As physicists, we often have a tendency to focus our classroom attentions solely on the content of the class - What should we cover and how? As physics educators, we often have a tendency to focus on what our students bring to the classroom that either does or does not match the way professionals think about a topic. But it is important to remember that physical laws are not given from the outside, they are the human brain's attempt to find ways to think about the physical universe that make sense to the human mind.

As we think about how to teach particular topics in physics, we need to consider what we might call: The Cognitive Physics Question - Does thinking about how people think change the way we choose to talk (and think) about physical laws and principles? 
When we consider how we teach physics, we can't just take everything we have learned and create a class that works for ourselves. We know so much more than our students - some of it being so deeply learned that we don't even realize we know it or are using it. When reading a story to a 4 or 5 year old with the goal of helping her begin to learn to read, we might not appreciate that the very idea that she might not have any idea that the symbols on the page represent the words you are saying. Do we have a similar problem with the physics we are trying to teach? Can understanding thinking and learning might help us better design learning goals for our courses.

18. Does understanding how people think and learn help us "deconstruct" our knowledge of physics so as to better understand what kinds of problems our students might have?

19. How does understanding something about how our students think and learn change the physics content we want to teach and how to orient it for them?

We already understand enough about thinking and learning to appreciate that learning physics is learning a way to think, not just learning facts about the physics. There is "content beyond the content," as well. Physics is not just about what are the laws about how the world works, it's about ways to think about how the world works and ways to find out new knowledge.

20. How can we teach more general way of thinking at the same time we are teaching specific content topics? What is particularly valuable for our students to know?

\section{DESIGNING ENVIRONMENTS}

As mentioned above, an essential goal of PER is figuring out how to teach physics more effectively. We can call this: The Engineering Question - How can we create learning environments that respond most effectively to help particular populations of students learn physics in a variety of situations?

This question is the one that has been most successfully addressed by the PER community and therefore we don't elaborate on it by adding specific questions. The outline of our future work can be seen in our past success as primarily a research-based design community (i.e., not a pure-research community). Researchers have studied specific issues in student learning. Curricula have been designed, implemented, evaluated, and refined. Issues of classroom design have been as important (though often less officially discussed) as indi- vidual learning. Thinking through the core concepts of the physics has led to excellent research and evaluation questions and has often led to researchers developing a deeper understanding of the material. Results have been presented at conferences and published in journals.[4] As a community, we understand these methods, agree on their relevance, and build on past history. We may each emphasize different elements, but we must keep in mind the interconnectedness of the research results when we ask ourselves, "What do I do in class on Monday?"

\section{CONCLUSION}

We are not suggesting that these questions have not been deeply researched in other areas that study human behavior. (See for example, [4].) Rather, we are suggesting that to begin to make more sense of what we do in PER, we will have to relate what we are learning to what is known in other fields, in general education research, in cognitive science, neuroscience, and sociology. We intend that these questions all be interpreted in the context of physics learning. A broad overall questions is: The Discipline Question - To what extent is thinking about and learning physics (and other scientific disciplines) different from the more general learning and thinking that tends to be studied in cognitive science and education research?

In the next decades we need to build on what is known, not only in our own field, but in other relevant research areas in order to make more sense of our own research so that we can truly begin to create a coherent and useful synthesis.

\section{ACKNOWLEDGMENTS}

This work is supported in part by a grant from the National Science Foundation (\#REC-0087519).

\section{REFERENCES}

[1] E. T. Whittaker, A History of Theories of Aether and Electricity (Dover Press, 1990).

[2] E. F. Redish, in Proceedings of the Enrico Fermi Summer School, Course CLVI, E. Redish and M. Vicentini, eds. (Italian Physical Society, 2004) pp. 1-64

[3] V. Otero, ibid. pp. 447-472.

[4] L. C. McDermott and E. F. Redish, Am. J. Phys., 67, pp. 755-767 (1998).

[5] J. Bransford, A. Brown, and R. Cocking, How People Learn: Brain, Mind, Experience, and School (National Academies Press, 2000). 